\title{
Income effects, cost damping and the value of time: theoretical properties embedded within practical travel choice models
}

\author{
Richard Batley ${ }^{1}$ (D)
}

Published online: 7 November 2016

(C) The Author(s) 2016. This article is published with open access at Springerlink.com

\begin{abstract}
Mackie et al. (Values of travel time savings in the UK. Report to Department for Transport. Institute for Transport Studies, University of Leeds \& John Bates Services, Leeds and Abingdon, 2003) proposed an identity relating the value of time (VoT) for commute and leisure travel to income and travel cost, reporting the prevalence of 'cost damping' (i.e. the phenomenon where VoT increases as travel cost increases). This identity (or a variant thereof) has been adopted within official methods for estimating VoT in the UK, Switzerland and The Netherlands. The present paper shows that Mackie et al.'s identity: (i) implies linear preferences, not strictly convex preferences as reported by Mackie et al.; (ii) complies with homogeneity and symmetry by construction; (iii) complies with adding-up if and only if VoT is unit elastic with respect to income; (iv) complies with negativity if VoT is unit elastic or greater with respect to income; (v) violates both addingup and negativity in the case of the 2003 UK national VoT study. We propose alternative identities which comply with adding-up and homogeneity by construction, and offer comparable fit to Mackie et al.'s identity on the UK VoT dataset. We also find that the imposition of adding-up and negativity on Mackie et al.'s identity, through appropriate constraint on model estimation, leads to an increase of around $20 \%$ in valuations from the 2003 UK dataset.
\end{abstract}

Keywords Value of time · Cost damping · Adding-up · Homogeneity · Negativity

JEL Classification B41 economic methodology · D01 microeconomic behaviour · Underlying principles

Richard Batley

R.P.Batley@its.leeds.ac.uk

1 Institute for Transport Studies (ITS), University of Leeds, Leeds LS2 9JT, UK 


\section{Introduction}

Within the context of a national study of the value of time (VoT) for non-work travel in the UK (i.e. covering commuting and leisure travel, but excluding travel in the conduct of an employer's business), Mackie et al. (2003, p. 29) proposed (in essence ${ }^{1}$ ) the following conditional indirect utility function (CIUF):

$$
\tilde{v}_{i, n}=\beta \cdot\left(\frac{y_{n}}{y_{0}}\right)^{\eta_{y}} \cdot\left(\frac{c_{n}}{c_{0}}\right)^{\eta_{c}} \cdot \Delta c_{i, n}+\alpha \cdot \Delta t_{i, n} \quad \text { for } i=1,2 ; n=1, \ldots, N
$$

where $^{2} \tilde{v}_{i, n}$ is the conditional indirect utility for travel option $i$ and traveller $n, y_{n}$ is the income of traveller $n, y_{0}$ is the 'reference' income (i.e. across a sample of travellers), $c_{n}$ is the 'current' travel cost for traveller $n, c_{0}$ is the 'reference' travel cost (i.e. across a sample of travel options), $\Delta c_{i, n}$ is the change in travel cost following an intervention, defined: $\Delta c_{i, n}=c_{i, n}-c_{n}$, $\Delta t_{i, n}$ is the change in travel time following an intervention, defined: $\Delta t_{i, n}=t_{i, n}-t_{n}, c_{i, n}$ is the 'design' travel cost for travel option $i$ and traveller $n$, (i.e. as presented within a Stated Preference (SP) design), $t_{i, n}$ is the 'design' travel time for travel option $i$ and traveller $n, t_{n}$ is the 'current' travel time for traveller $n$, and $\alpha, \beta, \eta_{y}, \eta_{c}$ are parameters to be estimated.

Since 2003, the CIUF (1) has underpinned official UK guidance for appraising the time saving benefits of transport infrastructure schemes (Department for Transport (DfT), 2014), and similar identities have subsequently been adopted within the official methods of other countries, notably Switzerland (Axhausen et al. 2008) and The Netherlands (Kouwenhoven et al. 2014). A key feature of (1) is that travel time and cost are specified as changes from the current travel option rather than as absolutes. This specification reflects the format of the dataset analysed by Mackie et al. (2003), which was collected by AHCG (1999) some years earlier, and consisted of SP choices between pairs of time/money changes relative to the current travel option. In order to estimate (1), the convention has been to deploy models from the Random Utility Model (RUM) class (e.g. McFadden 1981). However, since a property of RUM is 'translational invariance'-meaning that 'only differences in utility matter' (Train 2009, p. 19) — the empirical manifestation of (1) will be as follows:

$$
\tilde{v}_{i, n}=\beta \cdot\left(\frac{y_{n}}{y_{0}}\right)^{\eta_{y}} \cdot\left(\frac{c_{n}}{c_{0}}\right)^{\eta_{c}} \cdot c_{i, n}+\alpha \cdot t_{i, n} \quad \text { for } i=1,2 ; n=1, \ldots, N
$$

where $\Delta c_{i, n}$ and $\Delta t_{i, n}$ in (1) are, in effect, replaced with $c_{i, n}$ and $t_{i, n}$ respectively. When estimating (2) on SP data, it has commonly been found that $\partial \tilde{v}_{i, n} / \partial c_{i, n}<0$ and $\partial\left(\partial \tilde{v}_{i, n} / \partial c_{i, n}\right) / \partial c_{n}>0$, a result which has provoked debate concerning the phenomenon of 'cost damping'. In general terms, cost damping refers to instances where the conditional marginal disutility of travel cost decreases - in absolute terms - as travel cost and/or distance increases (Daly 2010). Within this general definition, different researchers have interpreted the phenomenon slightly differently, depending on their respective definitions of travel time, cost and distance. In the particular terms of the CIUF (2), we refer to cost damping as the propensity for the conditional marginal disutility of 'design' travel cost to decrease - in absolute terms - as 'current' travel cost increases.

\footnotetext{
${ }^{1}$ In practice, Mackie et al. adjusted the specification of travel time in (1) to account for 'size and sign' effects and 'inertia' effects; the latter phenomenon will be considered in the "Alternative specifications of the CIUF" section of the present paper, whilst the former falls outside the present scope.

${ }^{2}$ In what follows, $[\cdot]$ will be used to represent the arguments of a function and $(\cdot)$ to represent the collection of terms.
} 
Reconciling the empirical findings $\partial \tilde{v}_{i, n} / \partial c_{i, n}<0$ and $\partial\left(\partial \tilde{v}_{i, n} / \partial c_{i, n}\right) / \partial c_{n}>0$ with the theoretical properties of indifference maps, Mackie et al. (2003) concluded that '...the 'relative' effect whereby a given cost increase conveys less disutility when the base cost is large is not in line with general economic theory' (p. 29). Subsequently, Daly 2010 examined the same theoretical properties, but considering a broader class of CIUFs than (2), and concluded differently: '...economic theory cannot give any indication for the sign of the second derivative of indirect utility with respect to price... Since it is exactly the curvature of the utility function that defines cost damping, we see that economic theory is not able to give any indication for the existence or validity of cost damping' (p. 31). Whilst official guidance for appraising the time saving benefits of transport infrastructure schemes typically arises from a combination of theoretical and empirical insights, it would seem preferable if these two insights could be aligned-such that theory is supportive of empirical estimates of VoT, and vice versa. With this sentiment in mind, the present paper will reconsider the question of whether economic theory can shed light on the validity of cost damping, by examining the theoretical properties of demand functions as opposed to indifference maps. To this end, the specific objectives of the present paper are as follows:

- To consider the theoretical properties inherent within the CIUF (2), with particular reference to the cost damping phenomenon.

- To consider the theoretical properties of the conditional demand function associated with (2), and the extent to which these properties are affected by cost damping.

- To draw implications for the practical specification of travel choice models when estimating VoT.

- To illustrate these practical implications using an official national VoT dataset.

The paper will begin by briefly rehearsing the theoretical basis of VoT ("Theoretical basis of VoT" section), before analysing the first derivatives of (2), in particular deriving the VoT and the demand for travel conditional upon choice ("First derivatives from Mackie et al.'s CIUF" section). This will be followed by analysis of the second derivatives of (2), which inform the theoretical properties of both the indifference map ("Second derivatives from Mackie et al.'s CIUF, and theoretical properties of the indifference map" section) and the conditional demand ("Theoretical properties of Mackie et al.'s conditional demand" section). The penultimate section of the paper ("Alternative specifications of the CIUF" section) will test the properties of the conditional demand empirically using data from Mackie et al. (2003), whilst the final section will summarise and conclude ("Summary and conclusions" section).

\section{Theoretical basis of VoT}

Since Mackie et al. (2003) adopted De Serpa's (1971) maximisation problem as the theoretical basis for their VoT estimates, we will do likewise here. However, other conceptualisations of the problem pervade the literature, dating from the pioneering works of the 1960s and 1970s (e.g. Becker 1965; Oort 1969; Evans 1972), through to more recent works which have re-conceptualised and/or extended the maximisation problem (e.g. Jara-Díaz 2003; Castro et al. 2012). Accordingly, we assume that an individual' $\mathrm{s}^{3}$ unconditional

\footnotetext{
${ }^{3}$ In what follows, we will adopt some brevity in notation by suppressing the traveller index $n=1, \ldots, N$ and focussing upon a single individual. This should not be seen as a substantive loss of generality. Indeed, in the "Alternative specifications of the CIUF" section, we apply the model to a sample of travellers with different incomes and different travel times and costs for the current travel option.
} 
direct utility is a function of the quantity of goods consumed and the time spent in consuming these goods, subject to income, time and a 'time consumption' relationship between income and time. Although De Serpa considered various notions of time valuation, the present paper will focus upon the 'value of time as a commodity', which is given by the VoT spent consuming goods:

$$
v_{i}=\frac{\partial u / \partial t_{i}}{\lambda}=\frac{\mu-\kappa_{i}}{\lambda} \quad \text { for } i=1,2
$$

where $u$ is the unconditional direct utility, $\lambda$ is the marginal utility of income, $\mu$ is the marginal utility of time, and $\kappa_{i}$ is the marginal utility of saving time.

Applying (3) to the 'value of travel time' specifically, we adopt the standard proposition that the demand for travel derives from the demand for goods. Indeed, we can make the link between travel and consumption more explicit by assuming that:

- Goods 1 and 2 are in De Serpa's terms 'intermediate goods'; more specifically, let us assume that they are travel options.

- Prices and travel costs are one-and-the-same.

\section{First derivatives from Mackie et al.'s CIUF}

This section will consider the manner in which the value of time theory summarised in the "Theoretical basis of VoT" section relates to Mackie et al.'s (2003) empirical CIUF (2). More specifically, this section will derive three relevant quantities, namely the conditional marginal utility of travel cost, the conditional VoT, and the conditional demand for travel.

\section{Conditional marginal utility of travel cost}

For purposes of the theoretical analysis that follows, we assume $c_{0}=y_{0}=1$; this simplifies notation without imposing any substantive restriction on the generality of the analysis ${ }^{4}$. Assuming (rather strongly perhaps) that design cost is independent of current cost, the conditional marginal utility of travel cost in terms of design cost can be derived from (2):

$$
\frac{\partial \tilde{v}_{i}}{\partial c_{i}}=\beta \cdot y^{\eta_{y}} \cdot c^{\eta_{c}} \quad \text { for } i=1,2
$$

Note furthermore that (4) is equivalent to the conditional marginal utility of the change in travel cost derived from (1), i.e.

$$
\frac{\partial \tilde{v}_{i}}{\partial c_{i}}=\frac{\partial \tilde{v}_{i}}{\partial \Delta c_{i}} \quad \text { for } i=1,2
$$

Since the received wisdom is that travel cost is a 'bad', our a priori expectation is that $\beta<0$, in which case (4) would give rise to negative marginal utility, which might be otherwise referred to as marginal disutility.

\footnotetext{
${ }^{4}$ Mackie et al. (2003) noted that $c_{0}$ and $y_{0}$ are 'arbitrarily defined base or reference values which do not affect the estimation of the elasticities' (p. 30).
} 


\section{Conditional value of travel time}

Drawing reference to De Serpa (1971), the conditional VoT for the design journey, or equivalently the conditional value of the time/money changes, can be derived from (2) and (1) respectively ${ }^{5}$ :

$$
\tilde{v}_{i}\left[t_{i}, c_{i}\right]=\frac{\partial \tilde{v}_{i} / \partial t_{i}}{\partial \tilde{v}_{i} / \partial c_{i}}=\frac{\partial \tilde{v}_{i} / \partial \Delta t_{i}}{\partial \tilde{v}_{i} / \partial \Delta c_{i}}=\frac{\alpha}{\beta} \cdot y^{-\eta_{y}} \cdot c^{-\eta_{c}} \quad \text { for } i=1,2
$$

\section{Conditional demand for travel}

In an analogous manner to the conditional VoT (5), the CIUF (2) also allows-through application of Roy's identity-derivation of the conditional Marshallian demand for travel:

$$
\frac{\partial \tilde{v}_{i} / \partial c_{i}}{\partial \tilde{v}_{i} / \partial y}=-\frac{y}{\eta_{y} \cdot c_{i}}=\tilde{x}_{i}\left[c_{i}, y\right] \text { for } i=1,2
$$

Since $c_{i}, y \geq 0$ by definition, we can infer from (6) that:

- If $\eta_{y}<0$ then $\tilde{x}_{i}\left[c_{i}, y\right]>0$

- If $\eta_{y}>0$ then $\tilde{x}_{i}\left[c_{i}, y\right]<0$

In other words, the conditional demand for travel option $i$ will be non-negative-as required by theory-only where the elasticity of the conditional marginal utility of design cost with respect to income is negative (i.e. $\left.\eta_{y}<0\right)$. ${ }^{6}$ Note also that, where this elasticity is negative and unitary (i.e. $\eta_{y}=-1$ ), (6) will further to simplify to $y / c_{i}$, such that budget is fully exhausted and demand satiated.

\section{Second derivatives from Mackie et al.'s CIUF, and theoretical properties of the indifference map}

Building upon the working from the "First derivatives from Mackie et al.'s CIUF" section, the present section will take second derivatives of the CIUF (2), and then interpret these derivatives through reference to the theoretical properties of indifference maps. In this way, we will revisit the assertions of Mackie et al. (2003) and Daly (2010)-reported earlier in the "Introduction" section-concerning the theoretical properties of indifference maps and the consistency of these properties with the cost damping phenomenon.

\footnotetext{
5 Alternatively, and more faithful to De Serpa (1971), we could derive VoT for the design journey in terms of the conditional marginal utility of income rather than the conditional marginal utility of design cost:$$
\tilde{v}_{i}\left[t_{i}, y\right]=\frac{\partial \tilde{v}_{i} / \partial t_{i}}{\partial \tilde{v}_{i} / \partial y}=-\frac{\alpha \cdot y}{\eta_{y} \cdot \beta \cdot y^{\eta_{y}} \cdot c^{\eta_{c}} \cdot c_{i}} \quad \text { for } i=1,2
$$

This is directly proportional to Mackie et al.'s VoT (5), in the following manner:

$\tilde{v}_{i}\left[t_{i}, y\right]=\tilde{v}_{i}\left[t_{i}, c_{i}\right] \cdot \frac{y}{\eta_{y} \cdot c_{i}}=\tilde{v}_{i}\left[t_{i}, c_{i}\right] \cdot \tilde{x}_{i}\left[c_{i}, y\right]$ for $i=1,2$

The substantive distinction between the two measures is that (5) refers to a single trip, whereas (f2) refers to all trips by an individual. Also, the two measures have different signs since the denominator of (5) refers to a 'bad' (i.e. travel cost) whilst the denominator of (f2) refers to a 'good' (i.e. income).

${ }^{6}$ Derivation and interpretation of relevant elasticities from (2) will follow in the "Second derivatives from Mackie et al.'s CIUF, and theoretical properties of the indifference map" section.
} 


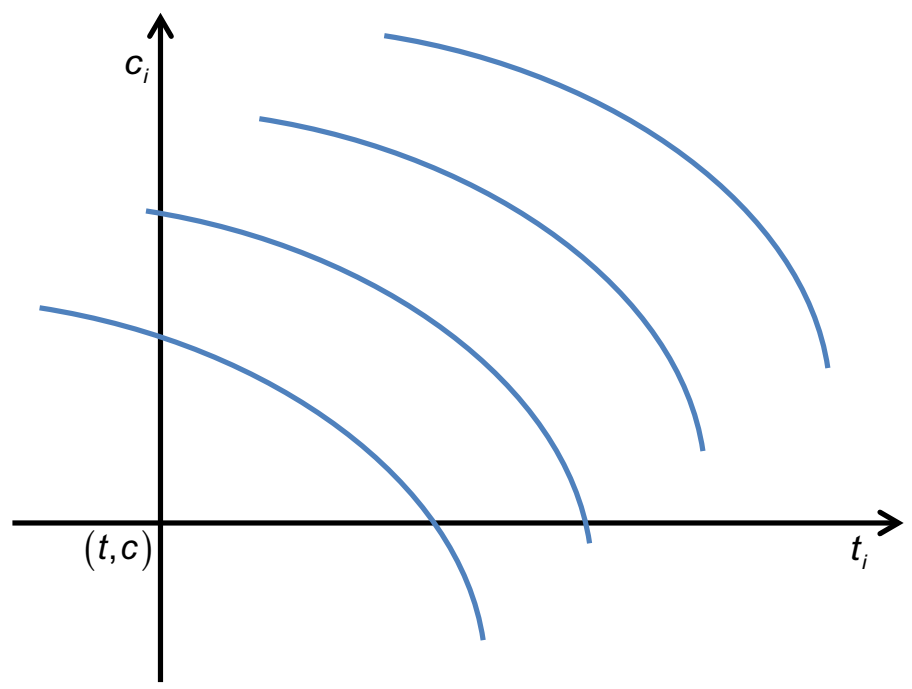

Fig. 1 Hypothesised indifference map based on design time and cost, for given current time and cost (This figure is adapted from Mackie et al. (2003), Fig. 1, p. 27)

The UK, Swiss and Dutch national VoT studies cited in the "Introduction" section all found the conditional marginal disutility of design cost (4) to decrease-in absolute termsas current cost increased. By implication, the conditional VoT for the design journey (5) was reported to increase as current cost increased. Although cost damping is frequently observed empirically (see Daly (2010) for a review of evidence), some researchers have argued that this phenomenon is inconsistent with microeconomic theory. Indeed, Mackie et al. (2003) expressed dissatisfaction with this finding in the UK VoT study, arguing that it violates 'strict concavity' - a well-behaved property of indifference curves.

Rationalising this argument, Mackie et al. presented a Lancaster-style (1966) indifference map (reproduced in Fig. 1 of the present paper) with design time on the horizontal axis, design cost on the vertical, and current time and cost at the origin; in other words, the indifference map was defined in terms of time and cost changes relative to the current travel option. It should however be qualified, that the identification issues inherent within the CIUF (1) imply that this indifference map could be centred on zero rather than $(t, c)$, without materially changing the empirical properties of the underpinning model. For the indifference map given in Fig. 1, Mackie et al. hypothesised that: 'On theoretical grounds, based on the expected shape of the indifference curves..., we would expect the marginal disutilities of both time and cost to increase as both time and cost increased, because of budget effects' (p. 27). In other words, Mackie et al. reasoned that we should expect to observe a decreasing marginal rate of substitution, wherein the conditional marginal disutility of design cost increases - in absolute terms - relative to the conditional marginal disutility of design time as design cost increases (and design time decreases, given the twodimensional nature of the indifference map) ${ }^{7}$, and vice versa. According to this rationale, the indifference map should embody strict concavity, such that the consumption of either 'bad' (i.e. design time/cost in excess of current time/cost) in isolation would be preferable to a combination of the two.

${ }^{7}$ That is to say: $\partial^{2} \tilde{v}_{i} / \partial t_{i}^{2}, \partial^{2} \tilde{v}_{i} / \partial c_{i}^{2} \leq 0$ for $i=1,2$. 
However, an important qualification should be issued which undermines the above rationale. Mackie et al.'s empirical results concerning cost damping were not based upon the second own partial derivatives of the CIUF (2) with respect to design cost-which is effectively the basis of Fig. 1-but rather upon the cross partial derivatives of the conditional marginal utility of design cost with respect to current cost. More specifically, Mackie et al. focussed upon the elasticities of the conditional marginal utility of design cost for options $i=1,2$ with respect to current cost;

$$
\frac{\partial\left(\partial \tilde{v}_{i} / \partial c_{i}\right)}{\partial c} \cdot \frac{c}{\left(\partial \tilde{v}_{i} / \partial c_{i}\right)}=\eta_{c} \cdot \beta_{c} \cdot y^{\eta_{y}} \cdot c^{\eta_{c}-1} \cdot \frac{c}{\left(\partial \tilde{v}_{i} / \partial c_{i}\right)}=\eta_{c} \quad \text { for } i=1,2
$$

and with respect to income:

$$
\frac{\partial\left(\partial \tilde{v}_{i} / \partial c_{i}\right)}{\partial y} \cdot \frac{y}{\left(\partial \tilde{v}_{i} / \partial c_{i}\right)}=\eta_{y} \cdot \beta_{c} \cdot y^{\eta_{y}-1} \cdot c^{\eta_{c}} \cdot \frac{y}{\left(\partial \tilde{v}_{i} / \partial c_{i}\right)}=\eta_{y} \quad \text { for } i=1,2
$$

With reference to (2), the CIUF specifies the conditional marginal utility of design time to be independent of both current cost and income, such that (7) and (8) can also be interpreted as the (negative of the) elasticities of the conditional VoT for the design journey with respect to current cost and income respectively. Estimating (2) on SP data, Mackie et al. reported the empirical results given in Table 1 for (7) and (8).

Continuing with our qualification of Mackie et al.'s rationale, the conditional marginal utility of design time within the CIUF (2) is similarly independent of current time. Since $\partial \tilde{v}_{i} / \partial t_{i}<0$ and $\partial \tilde{v}_{i} / \partial c_{i}<0$, whilst $\partial\left(\partial \tilde{v}_{i} / \partial t_{i}\right) / \partial t=0$ and $\partial\left(\partial \tilde{v}_{i} / \partial c_{i}\right) / \partial c>0$, Mackie et al. in practice found that design time and cost were 'bads', and that the conditional marginal disutility of design cost decreased-in absolute terms - relative to the conditional marginal disutility of design time as current cost increased (and current time decreased, again given the two-dimensional nature of the indifference map). Therefore, Mackie et al. observed the marginal rate of substitution between design time and cost to be increasing as current cost increased (and current time decreased), and vice versa. In other words, these empirical results contradict Mackie et al.'s theoretical hypothesis noted earlier in this section, and (in terms of the cross partial derivatives of the conditional marginal utility of design cost with respect to current cost, at least) imply strict convexity rather than strict concavity.

If instead, and more conventionally, we take second own partial derivatives of the CIUF (2) with respect to design cost, then cost damping is eliminated since $\partial^{2} \tilde{v}_{i} / \partial c_{i}^{2}=0$ for $i=1,2$. Another way of rationalising this is to note that the VoT for the design journey (5) - which also represents the marginal rate of substitution between design time and cost-is independent of design cost (and time) and thus gives rise to a linear indifference map (Fig. 2). The lesson of this section is that, when drawing inferences regarding the second derivatives of the CIUF (2), one needs to be clear concerning what dimensions the indifference map is defined on.

\section{Theoretical properties of Mackie et al.'s conditional demand}

Notwithstanding the preceding debate regarding the most defensible representation of the indifference map, strict concavity/convexity constitutes a 'well-behaved' (but unnecessary) property and not a definitive test of theoretical validity. The present section will therefore pursue a different tack, by assessing the theoretical validity of the elasticity estimates (7) and (8) - noting that the former is associated with Mackie et al.'s (2003) definition of cost 
Table 1 Elasticities of conditional marginal utility of design cost from Mackie et al. (2003)

\begin{tabular}{lcc}
\hline Elasticity with respect to & Commuting & Other non-work \\
\hline Current cost $\eta_{c}$ & $-0.42(9.08)$ & $-0.31(11.86)$ \\
Income $\eta_{y}$ & $-0.36(7.58)$ & $-0.16(5.49)$ \\
\hline
\end{tabular}

t-statistics given in parentheses

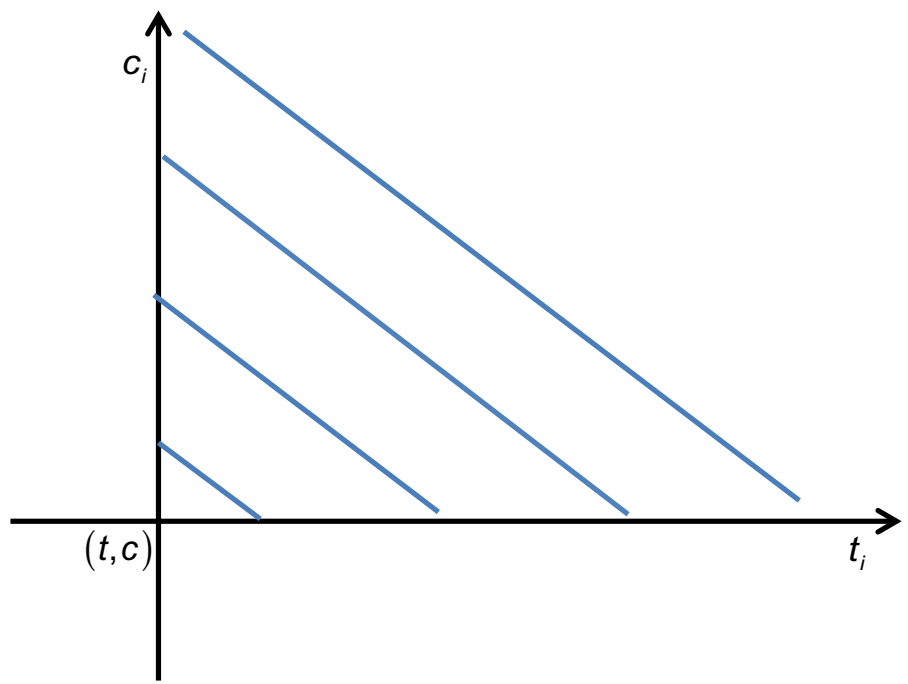

Fig. 2 Observed indifference map based on design time and cost, for given current time and cost

damping-in terms of the necessary and sufficient properties for the representation of the conditional demand; namely adding-up, homogeneity, negativity and symmetry ${ }^{8}$. As a prerequisite for this analysis, let us take the partial derivatives of the conditional Marshallian demand (6) with respect to design cost and income, respectively:

$$
\begin{array}{cc}
\frac{\partial \tilde{x}_{i}}{\partial c_{i}}=\frac{y}{\eta_{y} \cdot c_{i}^{2}} & \text { for } i=1,2 \\
\frac{\partial \tilde{x}_{i}}{\partial y}=-\frac{1}{\eta_{y} \cdot c_{i}} & \text { for } i=1,2
\end{array}
$$

Furthermore, if we rearrange the CIUF (2):

$$
y=\left(\frac{\tilde{v}_{i}-\alpha \cdot t_{i}}{\beta \cdot c^{\eta_{c}} \cdot c_{i}}\right)^{\frac{1}{\eta_{y}}} \quad \text { for } i=1,2
$$

then we can elicit, for a given level of conditional indirect utility, the conditional Hicksian demand via Shephard's Lemma:

\footnotetext{
${ }^{8}$ That is to say, if the Marshallian demand exhibits these properties then there exists a direct utility function which is maximised subject to a budget constraint. For fuller discussion of these properties in the context of continuous demand see Deaton and Muellbauer (1980), or in the context of discrete choice demand see Batley and Ibáñez (2013a).
} 


$$
\frac{\partial y}{\partial c_{i}}=-\frac{1}{\eta_{y}} \cdot(\cdot)^{\frac{1}{\eta_{y}}} \cdot \frac{1}{c_{i}}=\tilde{x}_{i}^{h} \quad \text { for } i=1,2
$$

where the notational abbreviation $(\cdot)=\left(\tilde{v}_{i}-\alpha \cdot t_{i}\right) /\left(\beta \cdot c^{\eta_{c}} \cdot c_{i}\right)$ is adopted, and the $h$ superscript distinguishes the Hicksian conditional demand from its Marshallian counterpart.

\section{Adding-up}

The adding-up property, which requires that the total value of demand is total expenditure, is pertinent to Mackie et al.'s mention of 'budget effects' (see earlier discussion in the “Second derivatives from Mackie et al.'s CIUF, and theoretical properties of the indifference map" section). If we express this property in terms of Engel and Cournot aggregations ${ }^{9}$, then we elicit the conditions (13a) and (13b) respectively, both of which must hold.

$$
\begin{gathered}
\frac{\partial \tilde{x}_{i}}{\partial c_{i}} \cdot c_{i}+\tilde{x}_{i}=0 \quad \text { for } i=1,2 \\
\frac{\partial \tilde{x}_{i}}{\partial y} \cdot c_{i}=1 \quad \text { for } i=1,2
\end{gathered}
$$

Substituting using (9) and (10) in (13a) and (13a), the adding-up conditions for the conditional demand (6) are given by (14a) and (14b):

$$
\begin{gathered}
\frac{\partial \tilde{x}_{i}}{\partial c_{i}} \cdot c_{i}+\tilde{x}_{i}=\frac{y \cdot c_{i}}{\eta_{y} \cdot c_{i}^{2}}-\frac{y}{\eta_{y} \cdot c_{i}} \quad \text { for } i=1,2 \\
\frac{\partial \tilde{x}_{i}}{\partial y} \cdot c_{i}=-\frac{1}{\eta_{y}} \quad \text { for } i=1,2
\end{gathered}
$$

Whilst Engel aggregation (14a) holds by definition, the only case where Cournot aggregation (14b) will hold is where $\eta_{y}=-1$ (i.e. where the conditional VoT is unit elastic with respect to income). ${ }^{10}$ However, since the estimate of $\eta_{y}$ in Table 1 is significantly different from minus one, for both commuting and other non-work, it can be concluded that Mackie et al.'s model violates adding-up.

\section{Homogeneity}

The homogeneity property requires that the Marshallian demands are homogenous of degree zero in both prices and income, as follows:

$$
\frac{\partial \tilde{x}_{i}}{\partial c_{i}} \cdot c_{i}+\frac{\partial \tilde{x}_{i}}{\partial y} \cdot y=0 \quad \text { for } i=1,2
$$

Again, this property is pertinent to Mackie et al.'s mention of budget effects (see the "Second derivatives from Mackie et al.'s CIUF, and theoretical properties of the indifference map" section above). Substituting using (9) and (10):

\footnotetext{
9 In the case of the conditional demand, however, there is no need to 'aggregate' as such.

${ }^{10}$ Whilst theory and evidence point to a temporal elasticity of VoT with respect to income of one (e.g. Fosgerau 2008), cross-sectional income elasticities estimated on SP data are usually somewhat less than one. In particular, Wardman and Abrantes (2011) reported an average income elasticity from meta-data of 0.5 .
} 


$$
\frac{\partial \tilde{x}_{i}}{\partial c_{i}} \cdot c_{i}+\frac{\partial \tilde{x}_{i}}{\partial y} \cdot y=\frac{y \cdot c_{i}}{\eta_{y} \cdot c_{i}^{2}}-\frac{y}{\eta_{y} \cdot c_{i}}=0 \quad \text { for } i=1,2
$$

It can thus be seen that Mackie et al.'s model complies with homogeneity by construction, irrespective of the sign and magnitude of $\eta_{y}$.

\section{Negativity}

The negativity property requires that the partial derivatives of the Hicksian demands with respect to own price are less than or equal to zero:

$$
\frac{\partial \tilde{x}_{i}^{h}}{\partial c_{i}} \leq 0 \quad \text { for } i=1,2
$$

Using (12) we can derive:

$$
\frac{\partial \tilde{x}_{i}^{h}}{\partial c_{i}}=\frac{1}{\eta_{y}} \cdot(\cdot)^{\frac{1}{\eta_{y}}} \cdot \frac{1}{c_{i}^{2}} \cdot\left(\frac{1}{\eta_{y}}+1\right)=-\frac{\tilde{x}_{i}^{h}}{c_{i}} \cdot\left(\frac{1}{\eta_{y}}+1\right) \quad \text { for } i=1,2
$$

where $(\cdot)$ is again a notational abbreviation from (11). Since $\tilde{x}_{i}^{h} \geq 0$ and $c_{i} \geq 0$ by definition, and the "Conditional demand for travel" section has shown that non-negative demand requires $\eta_{y}$ to be non-positive, two cases thus arise:

- If $-1<\eta_{y} \leq 0$, such that VoT increases with income but at a decreasing rate (i.e. is inelastic with respect to income), then negativity fails.

- If $\eta_{y} \leq-1$, such that VoT increases with income and at an increasing rate (i.e. is elastic with respect to income), then negativity holds.

Since the estimate of $\eta_{y}$ in Table 1 is significantly greater than minus one but significantly less than zero-for both commuting and other non-work-it can be concluded that Mackie et al.'s model violates negativity.

\section{Symmetry}

Finally, the symmetry property requires that the cross-price derivatives of the Hicksian demand should be symmetrical:

$$
\frac{\partial \tilde{x}_{1}^{h}}{\partial c_{2}}=\frac{\partial \tilde{x}_{2}^{h}}{\partial c_{1}}
$$

Since (12) is the conditional Hicksian demand, then by definition:

$$
\frac{\partial \tilde{x}_{1}^{h}}{\partial c_{2}}=\frac{\partial \tilde{x}_{2}^{h}}{\partial c_{1}}=0
$$

Therefore symmetry holds by construction.

\section{Alternative specifications of the CIUF}

The analysis in the preceding sections was based entirely upon Mackie et al.'s (2003) specification of the CIUF (2). In this, the penultimate section of the paper, we will repeat the same analysis for the alternative specifications given in Table 2, albeit in rather more 
succinct terms. More specifically, we will focus upon adding-up and homogeneity, since these properties would seem the most relevant to Mackie et al.'s interest in 'budget effects' (p. 27). The full set of specifications can be summarised as follows:

- Model I: As a benchmark against which the alternative specifications can be judged, this specification is the 'normalised' version of Mackie et al.'s CIUF, i.e. (2).

- Model II: This specification adopts a Cobb-Douglas form for income and design cost.

- Model III: This entails a linear 'residual income' specification, where residual income is expressed in terms of design cost.

- Model IV: This specification expresses design cost as a proportion of income, and introduces a power term on this ratio.

- Model V: This employs the same ratio as Model IV, but takes logarithms instead of specifying a power term. ${ }^{11}$

In a slightly different vein to Models I to $\mathrm{V}$, we also consider:

- Model VI: This specifies generalised relationships between each of the conditional marginal utilities that comprise VoT (i.e. $\alpha$ and $\beta$ ) and features of the journey/traveller (i.e. $c, t$ and $y$ ). The intention of Model VI is to mimic relationships reported in the most recent Danish national VoT study (Fosgerau et al. 2007), although it should be qualified that the Danish study specified models in VoT-space rather than utility-space ${ }^{12}$.

- Model VII: This is a simplified version of Model VI which specifies relationships between the $\beta$ parameter and features of the journey/traveller, but excludes similar relationships from the $\alpha$ parameter. This model might also be seen as a generalised version of the relationships inherent within Models I-V.

A number of other specifications were considered (notably specifications involving nonlinearity of income or residual income, so as to notionally introduce path dependence (Batley and Ibáñez 2013b)), but these could not be successfully estimated and are therefore omitted from the present discussion.

Considering first the explicit specifications (i.e. Models I-V), note that Model III fails adding-up by construction, whilst Models IV and V comply with both adding-up and homogeneity by construction. Models I and II are distinct in that theory confirms compliance with homogeneity, but compliance with adding-up is inconclusive and can be confirmed only through empirical analysis. ${ }^{13}$ Turning to the generalised specifications, Model VI fails both adding-up and homogeneity, whilst Model VII complies with addingup by construction and with homogeneity provided budget is exhausted (i.e. $\tilde{x}_{i}=y / c_{i}$ for $i=1,2$ ).

\footnotetext{
11 This specification corresponds to the non-linear-in-cost but linear-in-parameters form promoted by Rich and Mabit (2016).

12 Of course, models specified in VoT-space would not in practice identify the conditional marginal utilities of travel time and cost, and would not therefore lend themselves to testing in this manner.

13 See the "Adding-up" and "Homogeneity" sections for derivation of these theoretical properties for Model I, and the "Empirical analysis" and "Theoretical interpretation of the empirical analysis" sections for empirical testing of these properties for both Models I and II.
} 


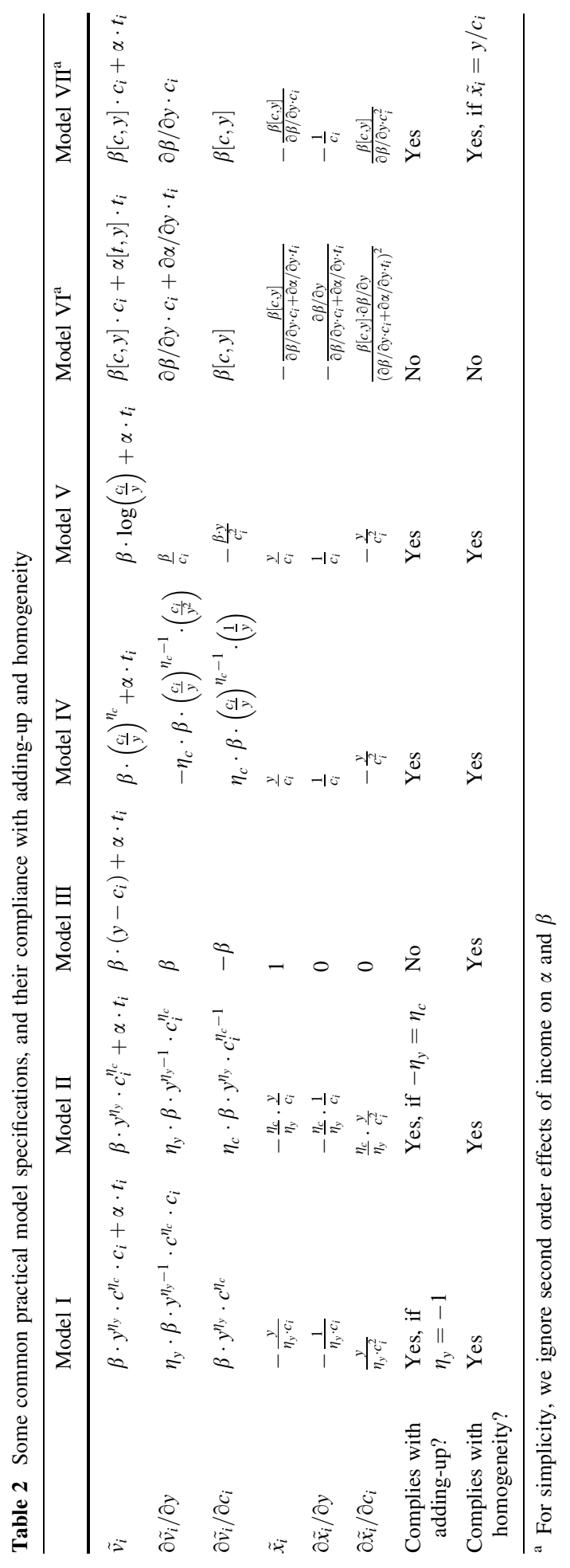




\section{Empirical analysis}

Without digressing into the philosophy of econometric method (e.g. Stigum 2003), we should acknowledge that the methodology of the present paper has thus far focussed upon the theoretical properties of model specifications for estimating VoT. The present section will now apply these models to the estimation of VoT on empirical data, further contributing to the method of the paper in two respects.

1. Empirical analysis will reveal whether, in the case of certain models, adding-up holds in practice.

2. Empirical analysis will reveal whether compliance with adding-up and homogeneity engenders models which better explain the empirical data vis-à-vis models which violate either or both of these properties.

Whilst such interests have not to date been a focus of either the VoT literature or the discrete choice modelling literature more generally, they have attracted considerable attention in the mainstream demand modelling literature; Deaton and Muellbauer's (1980) classic text—-though now slightly dated-gives a useful entry point to this literature.

As the basis for our empirical analysis, we employed the dataset collected in 1994 by Accent and Hague Consulting Group (AHCG), as part of a national study of non-work VoT commissioned by the UK DfT. This study designed and implemented several SP experiments, although here we will restrict attention to 'Experiment 1', which was focussed upon time/money trade-offs for car travellers. The dataset was initially analysed by AHCG, although DfT encountered difficulties in implementing AHCG's (1999) recommendations. The Institute for Transport Studies (ITS) at the University of Leeds and John Bates Services were therefore commissioned to re-analyse the dataset, and to re-issue recommendations (Mackie et al. 2003). The ITS Leeds/Bates recommendations subsequently informed the revision of official UK estimates for non-work VoT and, apart from minor updates during the interim, these recommendations have remained largely intact to the present day. The models estimated by Mackie et al. distinguished between the 'commuting' and 'other non-work' journey purposes (i.e. in the manner of Table 1) although, for reasons of brevity, we will concentrate on the former. Using this 'commuting' dataset, which consisted of 4737 observations, Models I to V above (i.e. the explicit specifications) were estimated, with one minor qualification as remarked upon earlier in footnote 1 . In accordance with Mackie et al. we appended the CIUFs of all models with an additive dummy variable $\left(I n e_{i}\right.$ for $\left.i=1,2\right)$, to represent instances where one of the SP choices was the same as the current travel option, i.e.

$$
\tilde{v}_{i}=\cdots+\gamma \cdot \text { Ine }_{i} \quad \text { for } i=1,2
$$

where

$$
\text { Ine }_{i}\left\{\begin{array}{cc}
=1 & \text { if } \Delta t_{i}, \Delta c_{i}=0 \\
=0 & \text { otherwise }
\end{array}\right.
$$

and $\gamma$ is a further parameter to be estimated. Following estimation, we derived the VoT in terms of the design journey (i.e. in the manner of (5)). All models were estimated using BIOGEME (Bierlaire 2003), and the results are summarised in Table 3. 


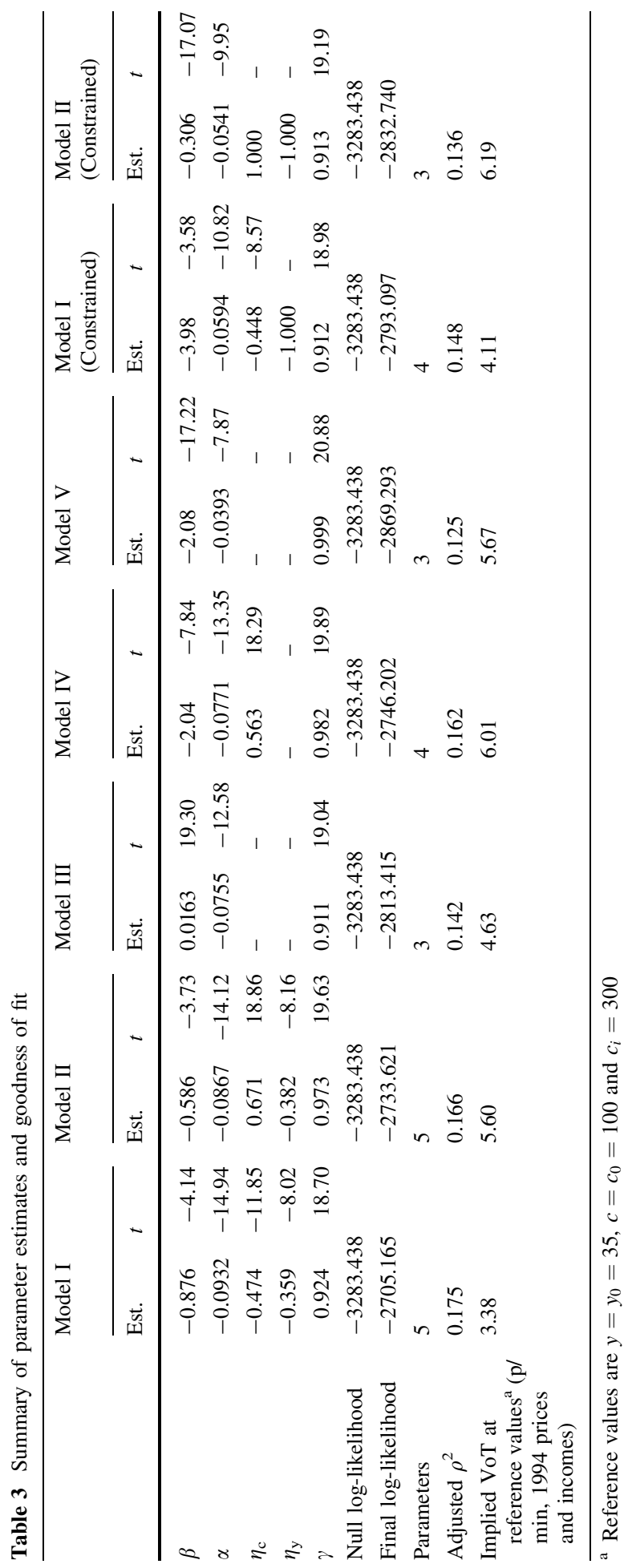




\section{Theoretical interpretation of the empirical analysis}

With reference to Table 3, note that Model I-which is ostensibly the model recommended by Mackie et al. (2003) ${ }^{14}$ - gives the best explanatory fit (in terms of the adjusted $\rho^{2}$ statistic) of the models considered, followed closely by Models II and IV. Models III and V give reduced fit, and this is possibly because the former excludes cost damping whilst the latter admits a restrictive form of cost damping, ${ }^{15}$ when the evidence from other models suggests that a more complex form of this phenomenon was prevalent in the dataset. Across all models, the estimated parameters carry their expected signs. Turning to the VoTs derived in terms of the design journey, Model I recommended by Mackie et al. yields the lowest valuation (3.38p/min, in 1994 prices and incomes), whilst Model IV yields the highest $(6.01 \mathrm{p} / \mathrm{min})$.

Focussing upon Models I and II in more detail, the theoretical properties of these models could not be confirmed a priori (see earlier discussion at the outset of the "Alternative specifications of the CIUF" section), but empirical analysis now reveals additional insights, as follows:

- Model I: Since $\eta_{y}$ is significantly different from minus one, we can infer that adding-up is violated. ${ }^{16}$

- Model II: Since $\eta_{y}$ is significantly different from $\eta_{\mathrm{c}}$, we can similarly infer that addingup is violated.

We can therefore conclude that, when applied to the AHCG dataset underpinning current official estimates of non-work VoT in the UK, the model recommended by Mackie et al. (Model I) violates adding-up. Note furthermore that the sign and magnitude of the $\eta_{\mathrm{c}}$ term influences compliance with adding-up only in the case of Model II, meaning that the cost damping phenomenon observed by Mackie et al. has no bearing on compliance in the case of Model I. In response to the above findings, we re-estimated Models I and II, but this time imposing the following constraints on estimation:

- Model I (Constrained): $\eta_{y}=-1$, thereby imposing adding-up. ${ }^{17}$

- Model II (Constrained): $-\eta_{y}=\eta_{c}=1$, again imposing adding-up. ${ }^{18}$

With reference to Table 3, these constraints ensure compliance with the theoretical properties of the conditional demand, but compromise (to some degree) explanatory fit. However, a notable outcome is that, for both Models I and II, the imposition of their respective constraints increases the VoT for the design journey; for example, in Model I recommended by Mackie et al. the implied VoT increases by around $20 \%$.

Finally, it is worth reflecting that Model IV offers superior fit to Models I and II (Constrained), accommodates cost damping in the form of second own partial derivatives of the CIUF with respect to design cost, and complies with adding-up and homogeneity by construction. This model would therefore seem to represent a credible alternative to Models I and II.

\footnotetext{
${ }^{14}$ Recalling earlier comment in footnote 1, the slight differences between the parameter estimates reported in Table 1 and for Model 1 in Table 2 reflect the omission of 'size and sign' effects in Table 2.

15 In Model $\mathrm{V}$, cost is damped in fixed proportion to design cost.

16 Based on the discussion within the "Negativity" section, it can also be inferred that negativity is violated.

17 Following from the "Negativity" section and footnote 16, this constraint also imposed negativity.

${ }^{18} \mathrm{We}$ also tried imposing the slightly weaker constraint $-\eta_{y}=\eta_{c}$, but this model could not be estimated.
} 


\section{Summary and conclusions}

A popular interest in transport demand modelling is to estimate the value of travel time (VoT), by implementing De Serpa's (1971) theory through empirical models from the random utility model (RUM) class. Within this framework, Mackie et al.'s (2003) conditional indirect utility function (CIUF) has proved influential, since this identity (or a variant thereof) has formed the basis of official national estimates of VoT in the UK, Switzerland and The Netherlands. A key feature of this CIUF, which was introduced as equation (1) in the present paper, is that it specifies utility as a function of changes in 'design' travel time and cost (i.e. for a given policy scenario such as an infrastructure scheme) from 'current' travel time and cost (i.e. in the absence of the scheme). However, when estimating as RUM, the property of 'translational invariance' means that the CIUF (1) is not identifiably different from one that specifies utility as a function of the absolute levels of design travel time and cost; the latter CIUF was introduced as equation (2) in the present paper. Estimation of (2) has commonly found that $\partial \tilde{v}_{i} / \partial c_{i}<0$ and $\partial\left(\partial \tilde{v}_{i} / \partial c_{i}\right) / \partial c_{n}>0$, such that the conditional marginal disutility of design cost decreases-in absolute terms - as current cost increases; this phenomenon is referred to as 'cost damping' in the literature.

Responding to the four objectives outlined in the "Introduction" section, the present paper has revealed the following insights on the CIUF (2):

- When estimating (2) in the course of the 2003 UK VoT study, Mackie et al. reported the implied indifference map to be strictly convex. We have argued that this result arises from an unconventional definition of the elasticity of VoT with respect to travel cost. Using a more conventional definition, the implied indifference map was shown to be linear rather than strictly convex.

- In an attempt to clarify the theoretical validity of the cost damping phenomenon, we assessed the compliance of the conditional demand implied by (2) with the theoretical properties of demand functions. We found that homogeneity and symmetry are imposed by construction, but adding-up holds only where the VoT is unit elastic with respect to income, and negativity holds where the same elasticity is unit elastic or greater.

- Relating these requirements to Mackie et al.'s empirical results, it was concluded that - in the case of the 2003 UK VoT study - the conditional demand violates both adding-up and negativity.

- The sign of $\partial\left(\partial \tilde{v}_{i} / \partial c_{i}\right) / \partial c_{n}$ had no bearing on the aforementioned violation of addingup and homogeneity. In fact, the determining factor was the sign (and magnitude) of $\partial\left(\partial \tilde{v}_{i} / \partial c_{i}\right) / \partial y$, i.e. the prevalence of cost damping with respect to income (rather than to travel cost).

- Repeating the same analysis with alternative specifications of the CIUF, we found that specifications which a) express design cost as a proportion of income, and b) introduce non-linearity through logarithmic transformation or through a power term, comply with the theoretical properties of demand functions. Comparing against (2) on Mackie et al.'s data, these specifications offered comparable explanatory power, whilst complying with adding-up and homogeneity. Furthermore, these specifications elicited higher valuations of travel time for the design journey.

- A final strand of analysis was to impose adding-up (and negativity) on Mackie et al.'s specification, through an appropriate constraint on model estimation. Whilst this 
constraint compromised explanatory fit to some degree, the implied VoT was found to increase by around $20 \%$.

Acknowledgements Thanks are due to Dr Manuel Ojeda Cabral, who estimated the models reported in Tables 2 and 3. An earlier version of this paper was presented at the International Association of Travel Behaviour Research Conference (Batley 2015), and the present version has benefitted from the comments of delegates at that conference, especially John Bates and Andrew Daly. Thanks are also due to two anonymous peer reviewers appointed by this journal, whose comments greatly improved the paper. Responsibility for any remaining faults or limitations rest entirely with the author.

Open Access This article is distributed under the terms of the Creative Commons Attribution 4.0 International License (http://creativecommons.org/licenses/by/4.0/), which permits unrestricted use, distribution, and reproduction in any medium, provided you give appropriate credit to the original author(s) and the source, provide a link to the Creative Commons license, and indicate if changes were made.

\section{References}

Abrantes, P.A.L., Wardman, M.R.: Meta-analysis of UK values of travel time: an update. Transp. Res. Part A 45(1), 1-17 (2011)

Accent \& Hague Consulting Group (AHCG) (1999) 'The value of travel time on UK roads'. Report to Department for Transport.

Axhausen, K.W., Hess, S., König, A., Abay, G., Bates, J.J., Bierlaire, M.: Income and distance elasticities of values of travel time savings: new Swiss results. Transp. Policy 15(3), 173-185 (2008)

Batley, R. (2015) Income effects, cost damping and the value of time: theoretical properties embedded within practical travel choice models. Paper presented at the International Association of Travel Behaviour (IATBR) Conference 2015, Windsor, UK.

Batley, R., Ibáñez, J.N.: Applied welfare economics with discrete choice models: the implications of theory for empirical specification. In: Hess, S., Daly, A. (eds.) Choice modelling: the state of the art and the state of practice, pp. 144-171. Edward Elgar, Cheltenham (2013a)

Batley, R., Ibáñez, J.N.: On the path independence conditions for discrete-continuous demand. J. Choice Model. 7, 13-23 (2013b)

Becker, G.S.: A theory of the allocation of time. Econ J 75(299), 493-517 (1965)

Bierlaire, M. (2003) 'BIOGEME: A free package for the estimation of discrete choice models'. Proceedings of the 3rd Swiss Transportation Research Conference, Ascona, Switzerland.

Castro, M., Bhat, C.R., Pendyala, R.M., Jara-Díaz, S.R.: Accommodating multiple constraints in the multiple discrete-continuous extreme value (MDCEV) choice model. Transp. Res. Part B 46(6), 729-743 (2012)

Daly, A. (2010) 'Cost damping in travel demand models'. Report of a study for the Department for Transport. Available at: http://www.rand.org/pubs/technical_reports/TR717.html

Deaton, A., Muellbauer, J.: Economics and Consumer Behavior. Cambridge University Press, Cambridge (1980)

Department for Transport (2014) 'TAG UNIT A1.3, User and Provider Impacts. Available at: https://www. gov.uk/government/uploads/system/uploads/attachment_data/file/313801/webtag-a13-user-providerimpacts-may2014.pdf

De Borger, B., Fosgerau, M.: The trade-off between money and travel time: A test of the theory of referencedependent preferences. J Urban Econ 64(1), 101-115 (2008)

De Serpa, A.: A theory of the economics of time. Econ J 81(324), 828-846 (1971)

Evans, A.: On the theory of the valuation and allocation of time. Scott. J. Polit. Econ. 19(1), 1-17 (1972)

Fosgerau, M.: Unit income elasticity of the value of travel time savings. Paper presented at the European Transport Conference 2005 (2005)

Fosgerau, M., Hjorth, K., Lyk-Jensen, S.: The Danish value of time study: results for experiment 1. Danish Transport Research Institute (2007)

Jara-Díaz, S.R.: On the goods-activities technical relations in the time allocation theory. Transportation 30(3), 245-260 (2003) 
Kouwenhoven, M., de Jong, G.C., Koster, P., van den Berg, V.A.C., Verhoef, E.T., Bates, J., Warffemius, P.M.J.: New values of time and reliability in passenger transport in The Netherlands. Res. Transp. Econ. 47, 37-49 (2014)

Lancaster, K.J.: A new approach to consumer theory. J. Polit. Econ.74(2), 132-157 (1966)

McFadden, D.: Econometric models of probabilistic choice. In: Manski, C., McFadden, D. (eds.) Structural Analysis of Discrete Data: With Econometric Applications, pp. 198-272. The MIT Press, Cambridge, Massachusetts (1981)

Mackie, P., Wardman, M., Fowkes, A.S., Whelan, G., Nellthorp, J., Bates J.J.: Values of travel time savings in the UK. Report to Department for Transport. Institute for Transport Studies, University of Leeds \& John Bates Services, Leeds and Abingdon (2003)

Oort, O.: The evaluation of travelling time. J. Transp. Econ. Policy 3(3), 279-286 (1969)

Rich, J., Mabit, S.: Cost damping and functional form in transport models. Transportation 43(5), 889-912 (2016)

Stigum, B.P.: Econometrics and the Philosophy of Economics: Theory-Data Confrontations in Economics. Princeton University Press, Princeton (2003)

Train, K.: Discrete Choice Methods with Simulation, 2nd edn. Cambridge University Press, Cambridge (2009)

Richard Batley is Professor of Transport Demand \& Valuation and Director of the Institute for Transport Studies (ITS) at the University of Leeds. With a disciplinary background in transport economics, Richard's specialist expertise covers two related areas: first, valuing qualitative aspects of travel (e.g. journey time, punctuality and comfort) in monetary terms, and second, forecasting the impacts of changes in these qualitative aspects on the demand for travel. Richard has operated mainly at the interface between academe and public policy. His work has been reported to senior public servants and politicians, and has been adopted within official policy and practitioner guidance issued to transport operators and scheme promoters. 